\title{
Efeitos do Treinamento Resistido na Lipoproteína de Baixa Densidade
}

\section{Effects of Resistance Training on Low Density Lipoprotein}

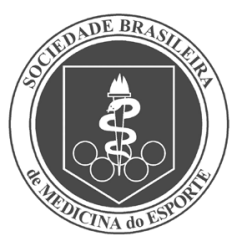

Artigo de Revisão

Jeferson Luis da Silva

Raul Cavalcante Maranhão

Carmen Guilherme Christiano de

Matos Vinagre

Laboratório de Metabolismo de Lípides - Instituto do Coração (Incor) da FMUSP - São Paulo - Brasil.

\section{Endereço para correspondência:}

Rua Dr. Enéas de Carvalho Aguiar, 44

Prédio II - 1으 Subsolo

05403-000 - São Paulo - SP

E-mail: jeferson.silva@incor.usp.br

\begin{abstract}
RESUMO
Os benefícios da prática regular do exercício físico estão claramente estabelecidos na literatura. Entretanto, a escolha do tipo de exercício ideal pode ser mais salutar para indivíduos com doenças específicas e patologias associadas. O propósito desta revisão foi verificar se o treinamento resistido (TR) exerce alguma alteração no colesterol da lipoproteína de baixa densidade (LDL-C). Foram observadas grandes diferenças na literatura, dificultando uma conclusão em relação aos benefícios do TR nesta revisão. No entanto, foi visto que o TR pode ser promissor na redução dos níveis de LDL-C, principalmente em homens e mulheres adultos, em pacientes com diabetes mellitus tipo 1 e tipo 2 e em mulheres pré-menopausa, não mostrando diferenças na população idosa. Os autores concluem que o TR é uma boa opção de exercício físico para indivíduos, principalmente quando o treinamento aeróbio (TA) é contraindicado.
\end{abstract}

Palavras-chave: exercício resistido, treinamento com pesos, LDL, colesterol.

\begin{abstract}
The benefits of exercise regular practice are clearly established in the literature. However, the choice of the ideal exercise may be more beneficial for individuals with specific diseases and associated pathologies. The aim of this review was to determine whether resistance training (RT) promotes any change on low density lipoprotein cholesterol. Important differences were observed in research protocols, making it difficult to define the benefits of RT in this review. However, it was noticed that RT may be promising in reducing LDL-C levels mainly in adult men and women, in patients with diabetes mellitus type 1 and type 2 and in pre-menopausal women, not presenting differences in the elderly population. It was concluded that the RT is an option good of physical exercise for individuals, especially when the aerobic training (AT) is contra-indicated.
\end{abstract}

Keyworks: resistance exercise, weight training, lipoprotein, cholesterol.

\section{INTRODUÇÃO}

A prática regular de atividade física através de grandes grupos musculares produz adaptações cardiovasculares que aumentam a capacidade de força da musculatura esquelética com exercício resistido. Essa prática regular previne o desenvolvimento de doença arterial coronária (DAC) e reduz sintomas em pacientes com doença cardiovascular estabelecida(1). O estilo de vida sedentário é associado ao aumento de doenças cardiovasculares. A falta de atividade física aumenta o estresse oxidativo, a disfunção endotelial e a aterosclerose ${ }^{(2)}$, sendo esta última a principal causa de morte em países ocidentais industrializados ${ }^{(3)}$. A maioria dos indivíduos que sofrem de doenças cardiovasculares tem um ou mais fatores de risco convencionais para a aterosclerose. Os fatores de risco incluem alterações na homocisteína, fibrinogênio, lipoproteínas, tamanho da partícula de lipoproteínas de baixa densidade (LDL) e proteína C-reativa ${ }^{(4)}$. O fator mais importante do processo de desenvolvimento da aterosclerose consiste na presença de concentrações plasmáticas elevadas de LDL-C ${ }^{(5)}$.

Em 1974, começaram a surgir os primeiros estudos relacionando exercício físico e lipoproteínas plasmáticas e a partir da década de 80 foram surgindo as primeiras evidências dos benefícios cardiovasculares do exercício físico. A IV Diretriz da Sociedade Brasileira de Cardiologia menciona o exercício físico regular como uma medida auxiliar para o controle das dislipidemias e tratamento da DAC (grau de recomendação I, nível de evidência A). A prática de exercícios físicos aeróbios promove redução dos níveis plasmáticos de triglicérides (TG) e aumento dos níveis de colesterol de lipoproteína de alta densidade $(\mathrm{HDL}-\mathrm{C})^{(6)}$. Entretanto, os efeitos do exercício físico na redução dos níveis plasmáticos de LDL-C ainda não são conclusivos. Em uma metanálise com 1.260 indivíduos portadores de doença cardiovascular não se observou alteração significativa nos níveis de LDL-C após a prática de exercícios aeróbios ${ }^{(7)}$, porém, em homens saudáveis houve tendência à redução(8) ${ }^{(8)}$ número de estudos que relatam os benefícios do treinamento resistido (TR) na redução do risco de doenças crônicas, na melhora das funções diárias e no aumento de força e massa muscular ${ }^{(9)}$ tem aumentado, porém, as conclusões sobre sua ação nas lipoproteínas plasmáticas ainda são controversas $^{(10)}$, devido ao pequeno número de estudos e a grande quantidade de fatores que podem influenciar no resultado, como nível inicial dos lípides, idade, duração e intensidade do treinamento, 
consumo máximo de oxigênio $\left(\mathrm{VO}_{2}\right.$ máx), peso corporal e percentual de gordura corporal(11). O objetivo desta revisão foi analisar a interferência do TR nas concentrações plasmáticas de LDL-C em diversos grupos de indivíduos.

\section{METODOLOGIA}

Foi realizada uma busca no banco de dados da Biblioteca Nacional de Medicina dos EUA (U.S. National Library of Medicine, incluindo a base de dados Medline e jornais científicos de biomedicina), em todas as línguas, utilizando as seguintes palavras-chave: 'resistance exercíse', 'resistance training,' 'weight training, 'weight lifting', 'power training', 'power lifting,' 'bodybuilder' associadas a 'low density lipoprotein'. O período pesquisado foi de 1950 até maio de 2009. Com as palavraschave 'resistance exercíse' e 'low density lipoprotein' foram encontrados 245 artigos; 'resistance training' e 'low density lipoprotein', 165 artigos; 'weight training' e 'low density lipoprotein', 404 artigos; 'weight lifting' e 'low density lipoprotein', 46 artigos; 'power training' e 'low density lipoprotein', 36 artigos; 'power lifting' e 'low density lipoprotein', cinco artigos; e, finalmente, 'bodybuilder' e 'low density lipoprotein', apenas um artigo. Nessa revisão, após análise de todos esses artigos, foram incluídos 89 artigos originais, de revisão e metanálise de estudos randomizados e não randomizados envolvendo exercício resistido combinado com exercício aeróbio e apenas exercício resistido, sendo todos diretamente relacionados com os níveis de LDL-C, tornando essa revisão abrangente e concisa. Não houve critério de exclusão por idade, sexo ou etnia.

\section{RESULTADOS}

Os resultados controversos da literatura mostram a dificuldade de comprovar a influência do TR sobre os níveis de LDL-C, e diferenças nos protocolos do estudo, como tipo de treinamento, sequência dos exercícios, duração, número de séries, número de repetições, intervalo de descanso entre os treinos, séries e exercícios, velocidade do movimento, tipos de contração, frequência semanal e evolução das cargas, são pertinentes. Abaixo estão descritos os tópicos dividindo os efeitos do treinamento físico de acordo com as modificações nos níveis plasmáticos de LDL-C.

\section{Estudos que não mostraram modificações nos níveis plas- máticos de LDL-C com o TR e TA}

Como podemos ver na tabela 1, alguns estudos não mostraram nenhuma diferença de LDL-C com o TR em fisioculturistas ${ }^{(12,13,14,15)}$, em indivíduos com fatores de risco cardiovascular ${ }^{(10)}$, em homens e mulheres hipertensas ${ }^{(16)}$, em crianças e adolescentes ${ }^{(17)}$, em indivíduos hipercolesterolêmicos ${ }^{(18)}$, em mulheres jovens ${ }^{(19,20)}$, obesas ${ }^{(21)}$ e idosas ${ }^{(22,23,24,25)}$, em homens idosos ${ }^{(22,23,25)}$ e obesos ${ }^{(26)} \operatorname{com}$ mais de um fator de risco de DAC. Também não foram observadas mudanças como efeito agudo do exercício resistido(27). O LDL-C também não se alterou em homens e mulheres idosas e obesas participantes de um programa de reabilitação após recente evento cardíaco, porém, houve acentuada diminuição da proporção LDL/HDL nessa população(28) e em homens saudáveis ${ }^{(29,30,31)}$. Em pacientes com insuficiência cardíaca crônica (ICC) não houve alteração no perfil lipídico após seis meses de TA e TR ${ }^{(32)}$. Em mulheres pós-menopausa, submetidas a TR leve associado a caminhada e dieta, não houve diferença no LDL-C comparando com grupos submetidos apenas a

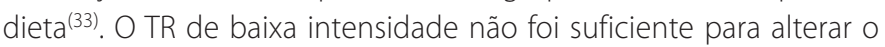
perfil de lípides em mulheres pós-menopausa após oito semanas(34) e em mulheres pós-menopausa com diabetes tipo $\|^{(35)}$ A maioria dos estudos com idosos não confirmou os benefícios do TR nos níveis de LDL-C (23,36), mesmo com variação de intensidade ${ }^{(37)}$ e com o treinamento utilizando o próprio peso do corpo $^{(38)}$. Ocorreu diminuição de LDL-C, porém, também diminuiu o HDL-C (39). Um estudo multicêntrico com 1.193 mulheres e 5.460 homens avaliou a relação das lipoproteínas com a força muscular, através de um exercício resistido de uma repetição máxima para região superior e outro para a região inferior do corpo; não encontrou associação entre a concentração de LDL-C e a força muscular entre homens e mulheres, observando apenas uma relação inversa, nos homens, entre a força e a HDL-C ${ }^{(40)}$.

\section{Estudos que mostraram aumento nos níveis plasmáticos de LDL-C com o TR e TA}

Comparando-se os níveis de LDL-C em fisiculturistas, corredores e levantadores de peso, observou-se que fisiculturistas e corredores apresentaram concentrações semelhantes da lipoproteína. Porém, os levantadores de peso tiveram aumento de LDL-C, o que sugere que esse foco de treinamento é menos favorável que em fisiculturista para o perfil de lípides ${ }^{(41)}$, apesar de ambas as modalidades utilizarem o TR. Além do tipo de TR, a alimentação pode influenciar de forma diferente no LDL-C. Nesse sentido, homens idosos e suplementados com proteína derivada da carne bovina submetidos a TR apresentaram maior concentração de LDL-C do que o grupo de homens vegetarianos treinados ${ }^{(42)}$. Em homens treinados, após cinco semanas de destreino, não houve alteração nos níveis de LDL-C ${ }^{(43)}$. Em outro estudo, as interrupções dos TR e TA por 14 dias não foram suficientes para alterar o perfil dos lípides em atletas, porém, houve diminuição do $\mathrm{VO}_{2}$ máx no grupo do TA e não houve alteração nos parâmetros de força no grupo do $\mathrm{TR}^{(44)}$. Contudo, o destreino por seis semanas reverteu as modificações benéficas causadas no perfil lipídico após um período de 16 semanas de TA em mulheres; no entanto, os parâmetros de força muscular continuaram presentes com o $T R^{(19)}$, mostrando que o efeito do TR nos lípides plasmáticos em homens pode persistir por no mínimo cinco semanas de destreino, o que não ocorre em mulheres, que com a interrupção do treinamento por seis semanas podem apresentar diminuição dos efeitos do exercício no perfil lipídico. Outro fato importante é a piora do perfil lipídico após um ano de destreino de TR e TA ${ }^{(43)}$ (tabela 3).

\section{Estudos que mostraram diminuição nos níveis plasmáticos de LDL-C com o TR e TA}

Como podemos ver na tabela 2, alguns estudos mostram diminuição da LDL-C associando TR com TA. Trabalhos que combinaram os dois tipos de treinamento mostraram melhora na redução de LDL-C em homens não obesos ${ }^{(45)}$, homens negros ${ }^{(46)}$, homens idosos ${ }^{(47)}$ e em homens e mulheres obesas com e sem dieta suplementada com fibra solúvel diminuíram os níveis de LDL-C ${ }^{(48)}$, mulheres com síndrome do ovário policístico (SOP) ${ }^{(49)}$ e adolescentes obesos com retardo mental ${ }^{(50)}$. Outro grupo beneficiado com essa combinação de treinamentos foi o de homens com paraplegia crônica, nos quais se observaram diminuição de LDL-C e decréscimo de cerca de $25 \%$ no risco de doença cardiovascular(51) (tabela 2). Além disso, o uso de fibras solúveis em indivíduos com fatores de risco cardiovascular associado a TR apresentou efeitos benéficos no perfil lipídico, com diminuição os níveis de LDL-C ${ }^{(52)}$. Alguns trabalhos observaram correlação entre menor taxa de LDL-C com baixa ingestão de alimentos com gordura e colesterol e também com baixa porcentagem de 
Tabela 1. Artigos pesquisados onde não há modificação no LDL-C com o TR e TA

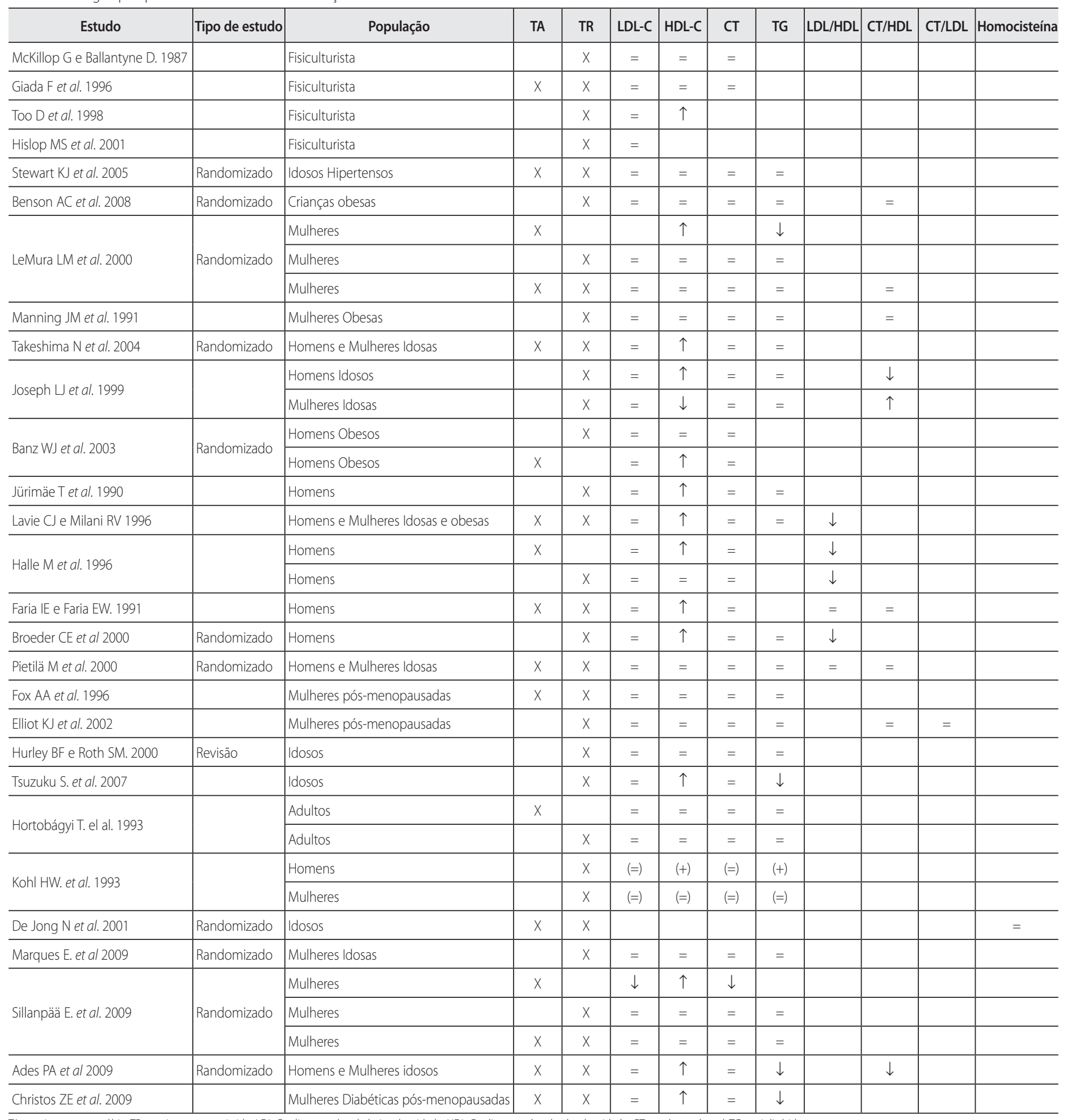

$\mathrm{TA}=$ treinamento aeróbio, $T R=$ treinamento resistido, $\mathrm{LDL}-\mathrm{C}=$ lipoproteína de baixa densidade, $\mathrm{HDL}-\mathrm{C}=$ lipoproteína de alta densidade, $\mathrm{CT}=$ colesterol total, $\mathrm{TG}=$ triglicérides.

gordura corporal em indivíduos treinados com TA e TR ${ }^{(53)}$. No entanto, mesmo com uma dieta rica em colesterol e gordura, indivíduos com alto índice de estresse por treinamento militar apresentaram redução de LDL-C em até $30 \%{ }^{(54)}$, assim como em atividades de alta intensidade ${ }^{(55)}$. No entanto, Tran e Weltman ${ }^{(56)}$ mostraram em uma metanálise que indivíduos treinados, que diminuem ou mantêm o peso corporal, apresentam redução nos níveis de LDL-C, enquanto que indivíduos que ganham peso têm o LDL-C aumentado. A relação peso corporal e LDL foi rejeitada em um estudo comparando fisiculturistas e indivíduos sem treino com o mesmo peso corporal; encontrou-se diminuição de LDL-C nos fisiculturistas ${ }^{(57)}$, mostrando que a composição corporal pode ser mais importante que o peso corporal. A diminuição do peso corporal promovida pela dieta em conjunto com o TA e o TR previne declínio normal da massa magra e da força muscular e melhora a composição corporal, a força máxima e o consumo máximo de oxigênio, comparado com perda de peso induzida apenas pela dieta(58). Após o ajuste da porcentagem de gordura corporal, a distribuição de gordura corporal e exercício físico têm papéis independentes em mulheres obesas ${ }^{(59) ;}$; tem sido demonstrado que mudanças na composição corporal de mulheres em pré-menopausa não se correlacionam com as alterações do colesterol total e do LDL-C(60). 
Tabela 2. Artigos pesquisados onde há diminuição do LDL-C com o TR e TA

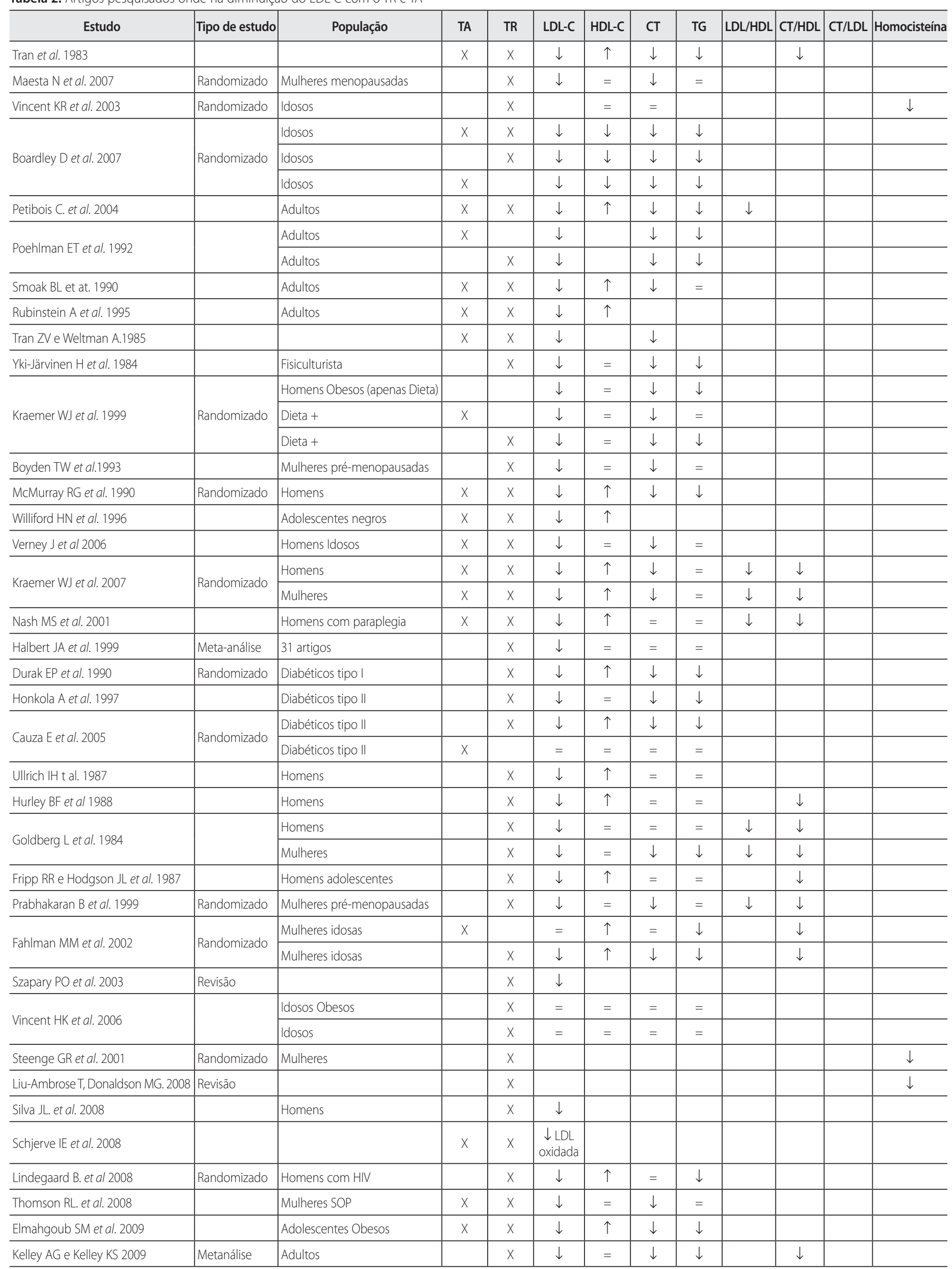

$T A=$ treinamento aeróbio, $T R=$ treinamento resistido, $L D L-C=$ lipoproteína de baixa densidade, $H D L-C=$ lipoproteína de alta densidade, $C T=$ colesterol total, $T G=$ triglicérides. 
Tabela 3. Artigos pesquisados onde há aumento de LDL-C com o TR e TA.

\begin{tabular}{|c|c|c|c|c|c|c|c|c|c|c|c|c|}
\hline Estudo & Tipo de estudo & População & TA & TR & LDL-C & HDL-C & CT & TG & LDL/HDL & $\mathrm{CT} / \mathrm{HDL}$ & $\mathrm{CT} / \mathrm{LDL}$ & Homocisteína \\
\hline Petibois C. et al. 2004 & & Adultos & $x$ & $x$ & $\uparrow$ & $\downarrow$ & $\uparrow$ & $\uparrow$ & $\uparrow$ & & & \\
\hline \multirow{3}{*}{ Hurley BF. et al. 1984} & & Levantadores de peso & & $x$ & $\uparrow$ & $\downarrow$ & & & $\uparrow$ & & & \\
\hline & & Fisiculturista & & $x$ & $=$ & $=$ & & & $=$ & & & \\
\hline & & Corredores & $x$ & & $=$ & $=$ & & & $=$ & & & \\
\hline \multirow{2}{*}{ Haub MD et al. 2005} & \multirow{2}{*}{ Randomizado } & $\begin{array}{l}\text { Homens idosos c/ Ingestão } \\
\text { de carne bovina }\end{array}$ & & $x$ & $\uparrow$ & $\uparrow$ & $\uparrow$ & $=$ & & $=$ & & \\
\hline & & $\begin{array}{l}\text { Homens idosos c/ Ingestão } \\
\text { de carne de soja }\end{array}$ & & $x$ & $=$ & $\downarrow$ & $=$ & $=$ & & $=$ & & \\
\hline
\end{tabular}

$T A=$ treinamento aeróbio, $T R=$ treinamento resistido, $L D L-C=$ lipoproteína de baixa densidade, $H D L-C=$ lipoproteína de alta densidade, $C T=$ colesterol total,$T G=$ triglicérides

\section{Estudos que mostraram modificações nos níveis plasmáti- cos de LDL-C apenas com o TR}

Estudos em populações diversas submetidas somente a TR mostraram diminuição significativa de LDL-C. Uma metanálise com 32 estudos mostrou diminuição de LDL-C no TR em indivíduos hiperlipidêmicos e normolipidêmicos( ${ }^{(61)}$. Em pacientes com diabetes mellitus tipo ${ }^{1(62)}$ e diabetes mellitus tipo $2^{(63,64)}$, houve diminuição relevante de LDL-C. TR com intensidade moderada e alto volume de exercícios, realizado em forma de circuito, tem se mostrado apropriado para indivíduos com diabetes mellitus tipo $2^{(63)}$. Diminuição significativa de LDL-C, apenas com o TR, também foi observada em homens ${ }^{(53,65,66,67)}$, mulheres ${ }^{(67)}$, homens adolescentes ${ }^{(68)}$, mulheres pré-menopausa ${ }^{(60,69)}$ e homens obesos ${ }^{(58)}$. Um interessante estudo com o TR de alta intensidade em mulheres idosas (70 a 87 anos) mostrou diminuição de LDL-C, mesmo sem alteração no peso e na dieta ${ }^{(70)}$. Um estudo randomizado com homens portadores de HIV com lipodistrofia reduziu o LDL-C, diminuiu a gordura corporal e melhorou a força muscular ${ }^{(71)}$. Uma metanálise com 95 estudos concluiu que o exercício físico reduz em $10,1 \%$ os níveis de LDL-C e a associação do exercício físico com dieta obteve redução de 8\% a 12 $\%$ de LDL-C(72). Outro estudo epidemiológico envolvendo mais de 50 empresas, com 8.499 homens que realizavam quatro a sete horas de TR por semana, mostrou que a alta qualidade do TR está fortemente associada à redução da incidência de hipercolesterolemia( ${ }^{(73)}$. Uma metanálise de ensaios controlados e randomizados selecionou 29 estudos representando 1.329 homens e mulheres adultos e mostrou que o treinamento resistido realizado de forma progressiva reduz 0 colesterol total (CT), a razão CT/HDL-C, a porção não HDL-C, os triglicérides (TG) e principalmente o LDL-C ${ }^{(74)}$.

\section{DISCUSSÃO}

O TR de alta intensidade pode diminuir o risco de doença arterial coronária independente do $\mathrm{VO}_{2}$ máx, peso e composição corporais em homens ${ }^{(66)}$. Além disso, alguns estudos mostraram que O TR aumenta $\mathrm{VVO}_{2}$ máx em homens ${ }^{(65)}$. Estudos que relacionaram o $\mathrm{VO}_{2}$ máx com lipoproteínas mostraram que homens com menor condicionamento físico $\left(\mathrm{VO}_{2}\right.$ máx $\left.<40 \mathrm{ml} / \mathrm{kg} / \mathrm{min}\right)$ e menor índice de aptidão física possuem um perfil desfavorável das subfrações de LDL e HDL, apresentando aumento da concentração de partículas de LDL pequenas e densas $(d>1,044 \mathrm{~g} / \mathrm{ml})$ e diminuição das partículas de HDL2a comparando-se com homens com um $\mathrm{VO}_{2}$ máx maior do que $50 \mathrm{ml} / \mathrm{kg} / \mathrm{min}$. Análise de regressão multivaria- da revelou que concentrações de LDL pequena e densa são determinadas principalmente pelo índice de massa corpórea (IMC), enquanto que de HDL2a e apolipoproteína A-I são determinadas principalmente pelo condicionamento físico $\left(\mathrm{VO}_{2}\right.$ máx $)$ em jovens homens saudáveis, sugerindo uma relação entre bom condicionamento físico, baixo peso corporal e perfil favorável da subfração da lipoproteína ${ }^{(75)}$. Em contrapartida, O’Donovan et al. ${ }^{(76)}$ relataram que o exercício físico não influencia o tamanho da partícula de LDL. No entanto, o treinamento físico realizado por longos períodos indica a formação de partículas de LDL com maior conteúdo de colesterol e pobres em proteína, sendo essa uma das formas pelas qual o exercício físico exerce um efeito protetor na doença cardiovascular ${ }^{(77)}$. Alguns dados sugerem que o TR pode diminuir o LDL-C, apesar de seus efeitos sobre os lípides ainda serem modestos e variáveis, promovendo importante redução da morbidade e mortalidade na população com doenças do coração, sendo especialmente importante para pacientes com dislipidemia aterogênica ${ }^{(78)}$. O efeito do exercício físico sobre os fatores de risco da aterosclerose pode ser significativamente ampliado por outras mudanças no estilo de vida, tais como modificações na composição da dieta e perda de peso, apesar de ser menor do que o promovido por terapias farmacológicas ${ }^{(1)}$

A concentração de homocisteína elevada pode determinar o risco de doença arterial coronária, através de alterações na função endotelial ${ }^{(79)}$, existindo uma correlação entre homocisteína e o LDL-C ${ }^{(80)}$. Os exercícios intensos e extenuantes têm demonstrado aumento nas espécies reativas de oxigênio (ERO), resultando em maior estresse oxidativo e possivelmente liberando fatores inflamatórios; isso ocorre principalmente através do overtraining (excesso de treinamento) ${ }^{(81)}$. No entanto, os efeitos crônicos do exercício resistido mostram uma regulação adaptativa do sistema antioxidante no miocárdio, sugerindo que sessões acumulativas de exercício resistido podem prevenir a peroxidação lipídica e preservar a capacidade antioxidante do miocárdio ${ }^{(82)}$. Vincent et al. ${ }^{(83)}$ observaram que o aumento de força muscular foi correlacionado com diminuição nos níveis de homocisteína após seis meses de $\operatorname{TR}^{(36,37,80,84)}$, enquanto que a mudança nos níveis de hidroperóxidos (PEROXs) foi correlacionada com a gordura corporal, mostrando que o TR reduz o estresse oxidativo e os níveis de homocisteína independente do adipócito, indicando proteção para idosos obesos e idosos saudáveis. Além disso, os autores sugerem que o TR pode oferecer proteção contra fatores de risco cardiovascular emergentes, através de exercícios físicos adequados 
ao peso corporal(83), podendo prevenir declínio cognitivo em idosos pela via do mecanismo de IGF-I e da homocisteína(85). Apenas um trabalho não mostrou diminuição da homocisteína em idosos após 17 semanas de treinamento físico(86).

Um importante efeito do TA foi demonstrado em avaliação do metabolismo da LDL através da utilização de uma nanoemulsão lipídica artificial que se liga a receptores de LDL. A remoção plasmática da nanoemulsão foi cinco vezes mais rápida em indivíduos com TA, provavelmente ocasionada por aumento de receptores de LDL ativados pelo exercício físico aeróbio(87). Resultados semelhantes foram encontrados em um estudo realizado com indivíduos treinados com $\mathrm{TR}^{\left({ }^{(8)}\right)}$, os quais tiveram aumento de três vezes na remoção plasmática da nanoemulsão. O menor tempo de permanência da LDL na circulação sanguínea diminui a chance de oxidação. Nesse sentido, os indivíduos com TA apresentaram menor concentração de LDL oxidada. Além disso, esses resultados obtidos tanto com TA como com TR devem estar relacionados com os efeitos antiaterogênicos do exercício físico. Em adição, um recente trabalho mostrou que o TR diminui a LDL oxidada, melhora a condição antioxidante, a função endotelial e a saúde cardiovascular, sendo uma excelente opção de exercício quando o TA for contraindicado ${ }^{(89)}$.

\section{REFERÊNCIAS BIBLIOGRÁFICAS}

1. Thompson PD, Buchner D, Pina IL, Balady GJ, Williams MA, Marcus BH, Berra K, et al. Exercise and physical activity in the prevention and treatment of atherosclerotic cardiovascular disease: a statement from the Council on Clinical Cardiology (Subcommittee on Exercise, Rehabilitation, and Prevention) and the Council on Nutrition, Physical Activity, and Metabolism (Subcommittee on Physical Activity). Circulation 2003;24;107:3109-16.

2. Laufs U, Wassmann S, Czech T, Münzel T, Eisenhauer M, Böhm M, Nickenig G. Physical inactivity increases oxidative stress, endothelial dysfunction, and atherosclerosis. Arterioscler Thromb Vasc Biol 2005;25:809-14

3. Ross R. The pathogenesis of atherosclerosis-an update. N Engl J Med 1986;20;314:488-500.

4. Kullo IJ, Ballantyne CM. Conditional risk factors for atherosclerosis. Mayo Clin Proc 2005;80:219-30.

5. Guyton A.C. Tratado de fisiologia médica. 8a ed. Rio de Janeiro: Guanabara Koogan, 1992.

6. Sposito AC, Caramelli B, Fonseca FA, Bertolami MC, Chacra AP, Maranhão RC, dos Santos RD, et al. IV Brazilian Guideline for Dyslipidemia and Atherosclerosis prevention: Department of Atherosclerosis of Brazilian Society of Cardiology. Arq Bras Cardiol 2007;88(supl 1):2-19.

7. Kelley GA, Kelley KS, Franklin B. Aerobic exercise and lipids and lipoproteins in patients with cardiovascular disease. J Cardiopulm Rehabil 2006;26:131-44.

8. Kelley GA, Kelley KS. Aerobic exercise and lipids and lipoproteins in men: a meta-analysis of randomized controlled trials. J Mens Health Gend 2006;3:61-70.

9. Phillips SM. Resistance exercise: good for more than just Grandma and Grandpa's muscles. Appl Physiol Nutr Metab 2007;32:1198-205.

10. Hurley BF. Effects of resistive training on lipoprotein-lipid profiles: a comparison to aerobic exercise training. Med Sci Sports Exerc 1989;21:689-93.

11. Tran ZV, Weltman A, Glass GV, Mood DP. The effects of exercise on blood lipids and lipoproteins: a meta-analysis of studies. Med Sci Sports Exerc 1983;15:393-402.

12. McKillop G, Ballantyne D. Lipoprotein analysis in bodybuilders. Int J Cardiol 1987;17:281-8.

13. Giada F, Zuliani G, Baldo-Enzi G, Palmieri E, Volpato S, Vitale E, Fellin R, et al. Lipoprotein profile, diet and body composition in athletes practicing mixed an anaerobic activities. J Sports Med Phys Fitness 1996;36:211-6.

14. Too D, Wakayama EJ, Locati LL, Landwer GE. Effect of a precompetition bodybuilding diet and training regimen on body composition and blood chemistry. J Sports Med Phys Fitness 1998;38:245-52.

15. Hislop MS, St Clair Gibson A, Lambert MI, Noakes TD, Marais AD. Effects of androgen manipulation on postprandial triglyceridaemia, low-density lipoprotein particle size and lipoprotein(a) in men Atherosclerosis 2001;159:425-32.

16. Stewart KJ, Bacher AC, Turner K, Lim JG, Hees PS, Shapiro EP, Ouyang P, et al. Exercise and risk factors associated with metabolic syndrome in older adults. Am J Prev Med 2005;28:9-18.

17. Benson AC, Torode ME, Fiatarone Singh MA. The effect of high-intensity progressive resistance training on adiposity in children: a randomized controlled trial. Int J Obes (Lond) 2008;32:1016-27.

18. Warber J, Bazzarre T. A comparison between running and weight lifting on fasting plasma lipids of a well-conditioned hypercholesterolemic male. Int J Sport Nutr 1991;1:265-78.

19. LeMura LM, von Duvillard SP, Andreacci J, Klebez JM, Chelland SA, Russo J. Lipid and lipoprotein profiles, cardiovascular fitness, body composition, and diet during and after resistance, aerobic and combination training in young women. Eur J Appl Physiol 2000;82:451-8.

\section{CONCLUSÃO}

Em conclusão, resultados conflitantes dos efeitos do TR sobre o LDL-C mostram a necessidade de mais estudos com protocolos padronizados e randomizados, o que evitaria a interferência de diversos fatores que possam prejudicar a interpretação dos dados. No entanto, a revisão bibliográfica sugere que o TR pode ser promissor na redução dos níveis de LDL-C principalmente em homens e mulheres adultos, em pacientes com diabetes mellitus tipo I e tipo II e em mulheres pré-menopausa, mas não em população idosa. Indivíduos com prédisposição para DAC podem ser beneficiados com o TR, diminuindo os riscos de aterosclerose, pela diminuição dos seus fatores, sendo os níveis de LDL-C o principal deles. Outro importante fator além da redução do LDL-C é a possível modificação na estrutura dessa lipoproteína e no número de seus receptores, que podem explicar os benefícios do exercício. Novos estudos randomizados e controlados são de fundamental importância para o entendimento dos mecanismos que envolvem o metabolismo da LDL com o TR.

Todos os autores declararam não haver qualquer potencial conflito de interesses referente a este artigo.

20. Sillanpää E, Laaksonen DE, Häkkinen A, Karavirta L, Jensen B, Kraemer WJ, Häkkinen K, et al. Body composition, fitness, and metabolic health during strength and endurance training and their combination in middle-aged and older women. Eur J Appl Physiol 2009;106:285-96.

21. Manning JM, Dooly-Manning CR, White K, Kampa I, Silas S, Kesselhaut M, Ruoff M. Effects of a resistive training program on lipoprotein-lipid levels in obese women. Med Sci Sports Exerc 1991;23:1222-6.

22. Takeshima N, Rogers ME, Islam MM, Yamauchi T, Watanabe E, Okada A. Effect of concurrent aerobic and resistance circuit exercise training on fitness in older adults. Eur J Appl Physiol 2004;93:173-82.

23. Joseph LJ, Davey SL, Evans WJ, Campbell WW. Differential effect of resistance training on the body composition and lipoprotein-lipid profile in older men and women. Metabolism 1999;48:1474.

24. Marques E, Carvalho J, Soares JM, Marques F, Mota J. Effects of resistance and multicomponent exercise on lipid profiles of older women. Maturitas 2009;20;63:84-8.

25. Ades PA, Savage PD, Toth MJ, Harvey-Berino J, Schneider DJ, Bunn JY, Ludlow M, et al. High-calorieexpenditure exercise: a new approach to cardiac rehabilitation for overweight coronary patients. Circulation 2009;26;119:2671-8..

26. Banz WJ, Maher MA, Thompson WG, Bassett DR, Moore W, Ashraf M, Zemel MB, et al. Effects of resistance versus aerobic training on coronary artery disease risk factors. Exp Biol Med (Maywood). 2003;228:434-40

27. Jürimäe $T$, Karelson $K$, Smirnova T, Viru A. The effect of a single-circuit weight-training session on the blood biochemistry of untrained university students. Eur J Appl Physiol Occup Physiol 1990;61:344-8.

28. Lavie CJ, Milani RV. Effects of cardiac rehabilitation and exercise training in obese patients with coronary artery disease. Chest 1996;109:52-6.

29. Halle M, Berg A, von Stein T, Baumstark MW, König D, Keul J. Lipoprotein(a) in endurance athletes, power athletes, and sedentary controls. Med Sci Sports Exerc 1996;28:962-6.

30. Faria IE, Faria EW. Effect of exercise on blood lipid constituents and aerobic capacity of fire fighters. J Sports Med Phys Fitness 1991;31:75-81.

31. Broeder CE, Quindry J, Brittingham K, Panton L, Thomson J, Appakondu S, Yarlagadda C, et al. The Andro Project: physiological and hormonal influences of androstenedione supplementation in men 35 to 65 years old participating in a high-intensity resistance training program. Arch Intern Med 2000;160:3093-104.

32. Pietilä M, Malminiemi K, Huupponen R, Rouru J, Pulkki K, Pere E, Voipio-Pulkki LM. Celiprolol augments the effect of physical exercise on insulin sensitivity and serum lipid levels in chronic heart failure. Eur J Heart Fail 2000;2:81-90

33. Fox AA, Thompson JL, Butterfield GE, Gylfadottir U, Moynihan S, Spiller G. Effects of diet and exercise on common cardiovascular disease risk factors in moderately obese older women. Am J Clin Nutr 1996;63:225-33.

34. Elliott KJ, Sale C, Cable NT. Effects of resistance training and detraining on muscle strength and blood lipid profiles in postmenopausal women. Br J Sports Med 2002;36:340-4.

35. Christos ZE, Tokmakidis SP, Volaklis KA, Kotsa K, Touvra AM, Douda E, Yovos IG. Lipoprotein profile, glycemic control and physical fitness after strength and aerobic training in post-menopausal women with type 2 diabetes. Eur J Appl Physiol 2009;106:901-7.

36. Hurley BF, Roth SM. Strength training in the elderly: effects on risk factors for age-related diseases. Sports Med 2000;30:249-68. 
37. Vincent KR, Braith RW, Bottiglieri T, Vincent HK, Lowenthal DT. Homocysteine and lipoprotein levels following resistance training in older adults. Prev Cardiol 2003;6:197-203.

38. Tsuzuku S, Kajioka T, Endo H, Abbott RD, Curb JD, Yano K. Favorable effects of non-instrumental resistance training on fat distribution and metabolic profiles in healthy elderly people. Eur J Appl Physiol 2007;99:549-55.

39. Boardley D, Fahlman M, Topp R, Morgan AL, McNevin N. The impact of exercise training on blood lipids in older adults. Am J Geriatr Cardiol 2007;16:30-5.

40. Kohl HW 3rd, Gordon NF, Scott CB, Vaandrager H, Blair SN. Musculoskeletal strength and serum lipid levels in men and women. Med Sci Sports Exerc 1992;24:1080-7.

41. Hurley BF, Seals DR, Hagberg JM, Goldberg AC, Ostrove SM, Holloszy JO, Wiest WG, Goldberg AP. High-density-lipoprotein cholesterol in bodybuilders v powerlifters. Negative effects of androgen use. JAMA 1984;252:507-13.

42. Haub MD, Wells AM, Campbell WW. Beef and soy-based food supplements differentially affect serum lipoprotein-lipid profiles because of changes in carbohydrate intake and novel nutrient intake ratios in older men who resistive-train. Metabolism 2005;54:769-74.

43. Petibois C, Cassaigne A, Gin H, Déléris G. Lipid profile disorders induced by long-term cessation of physical activity in previously highly endurance-trained subjects. J Clin Endocrinol Metab 2004;89:3377-84.

44. Hortobágyi T, Houmard JA, Israel RG, Carpenter JW, Heath J, Barakat HA. Effects of exercise cessation on lipids and lipoproteins in distance runners and power athletes. Eur J Appl Physiol Occup Physiol 1993;67:226-30.

45. McMurray RG, Harrell JS, Griggs TR. A comparison of two fitness programs to reduce the risk of coronary heart disease in public safety officers. J Occup Med 1990;32:616-20.

46. Williford HN, Blessing DL, Duey WJ, Barksdale JM, Wang N, Olson MS, Teel S. Exercise training in black adolescents: changes in blood lipids and $\mathrm{VO}_{2}$ max. Ethn Dis 1996;6:279-85.

47. Verney J, Kadi F, Saafi MA, Piehl-Aulin K, Denis C. Combined lower body endurance and upper body resistance training improves performance and health parameters in healthy active elderly. Eur J Appl Physiol 2006;97:288-97.

48. Kraemer WJ, Vingren JL, Silvestre R, Spiering BA, Hatfield DL, Ho JY, Volek JS, et al. Effect of adding exercise to a diet containing glucomannan. Metabolism 2007;56:1149-58.

49. Thomson RL, Buckley JD, Noakes M, Clifton PM, Norman RJ, Brinkworth GD. The effect of a hypocaloric diet with and without exercise training on body composition, cardiometabolic risk profile, and reproductive function in overweight and obese women with polycystic ovary syndrome. J Clin Endocrinol Metab 2008:93:3373-80.

50. Elmahgoub SM, Lambers S, Stegen S, Van Laethem C, Cambier D, Calders P. The influence of combined exercise training on indices of obesity, physical fitness and lipid profile in overweight and obese adolescents with mental retardation. Eur J Pediatr 2009;168:1327-33.

51. Nash MS, Jacobs PL, Mendez AJ, Goldberg RB. Circuit resistance training improves the atherogenic lipid profiles of persons with chronic paraplegia. J Spinal Cord Med 2001;24:2-9.

52. Maesta N, Nahas EA, Nahas-Neto J, Orsatti FL, Fernandes CE, Traiman P, Burini RC. Effects of soy protein and resistance exercise on body composition and blood lipids in postmenopausal women. Maturitas 2007;20;56:350-8.

53. Poehlman ET, Gardner AW, Ades PA, Katzman-Rooks SM, Montgomery SM, Atlas OK, Ballor DL, et al. Resting energy metabolism and cardiovascular disease risk in resistance-trained and aerobically trained males. Metabolism 1992;41:1351-60.

54. Smoak BL, Norton JP, Ferguson EW, Deuster PA. Changes in lipoprotein profiles during intense military training. J Am Coll Nutr 1990;9:567-72.

55. Rubinstein A, Burstein R, Lubin F, Chetrit A, Dann EJ, Levtov O, Dolev E, et al. Lipoprotein profile changes during intense training of Israeli military recruits. Med Sci Sports Exerc 1995;27:480-4.

56. Tran ZV, Weltman A. Differential effects of exercise on serum lipid and lipoprotein levels seen with changes in body weight. A meta-analysis. JAMA 1985;254:919-24.

57. Yki-Järvinen H, Koivisto VA, Taskinen MR, Nikkilä E. Glucose tolerance, plasma lipoproteins and tissue lipoprotein lipase activities in body builders. Eur J Appl Physiol Occup Physiol 1984;53:253-9.

58. Kraemer WJ, Volek JS, Clark KL, Gordon SE, Puhl SM, Koziris LP, Mcbride JM, et al. Influence of exercise training on physiological and performance changes with weight loss in men. Med Sci Sports Exerc 1999;31:1320-9.

59. Tanaka H, Kakiyama T, Takahara K, Yamauchi M, Tanaka M, Sasaki J, Shindo M, et al. The association among fat distribution, physical fitness, and the risk factors of cardiovascular disease in obese women. Obes Res 1995;3(Suppl 5):649S-53S.

60. Boyden TW, Pamenter RW, Going SB, Lohman TG, Hall MC, Houtkooper LB, Aickin M, et al. Resistance exercise training is associated with decreases in serum low-density lipoprotein cholesterol levels in premenopausal women. Arch Intern Med 1993;153:97-100.

61. Halbert JA, Silagy CA, Finucane P, Withers RT, Hamdorf PA. Exercise training and blood lipids in hyperlipidemic and normolipidemic adults: a meta-analysis of randomized, controlled trials. Eur J Clin Nutr 1999;53:514-22.

62. Durak EP, Jovanovic-Peterson L, Peterson CM. Randomized crossover study of effect of resistance training on glycemic control, muscular strength, and cholesterol in type I diabetic men. Diabetes Care 1990;13:1039-43.

63. Honkola A, Forsén T, Eriksson J. Resistance training improves the metabolic profile in individuals with type 2 diabetes. Acta Diabetol 1997;34:245-8.

64. Cauza E, Hanusch-Enserer U, Strasser B, Ludvik B, Metz-Schimmerl S, Pacini G, Haber P, et al. The relative benefits of endurance and strength training on the metabolic factors and muscle function of people with type 2 diabetes mellitus. Arch Phys Med Rehabil 2005;86:1527-33.

65. Ullrich $I H$, Reid CM, Yeater RA. Increased HDL-cholesterol levels with a weight lifting program. South Med J 1987;80:328-31.

66. Hurley BF, Hagberg JM, Goldberg AP, Seals DR, Ehsani AA, Brennan RE, Holloszy JO. Resistive training can reduce coronary risk factors without altering $\mathrm{VO}_{2} \max$ or percent body fat. Med Sci Sports Exerc 1988:20:150-4.

67. Goldberg L, Elliot DL, Schutz RW, Kloster FE. Changes in lipid and lipoprotein levels after weight training. JAMA 1984;252:504-6.

68. Fripp RR, Hodgson JL. Effect of resistive training on plasma lipid and lipoprotein levels in male adolescents. J Pediatr 1987;111:926-31.

69. Prabhakaran B, Dowling EA, Branch JD, Swain DP, Leutholtz BC. Effect of 14 weeks of resistance training on lipid profile and body fat percentage in premenopausal women. Br J Sports Med 1999;33:190-5.

70. Fahlman MM, Boardley D, Lambert CP, Flynn MG. Effects of endurance training and resistance training on plasma lipoprotein profiles in elderly women. J Gerontol A Biol Sci Med Sci 2002;57:B54-60.

71. Lindegaard B, Hansen T, Hvid T, van Hall G, Plomgaard P, Ditlevsen S, Gerstoft J, Pedersen BK. The effect of strength and endurance training on insulin sensitivity and fat distribution in human immunodeficiency virus-infected patients with lipodystrophy. J Clin Endocrinol Metab 2008:93:3860-9.

72. Shephard RJ, Balady GJ. Exercise as cardiovascular therapy. Circulation 1999;99:963-72.

73. Tucker LA, Silvester $\sqcup$. Strength training and hypercholesterolemia: an epidemiologic study of 8499 employed men. Am J Health Promot 1996;11:35-41.

74. Kelley GA, Kelley KS. Impact of progressive resistance training on lipids and lipoproteins in adults: a meta-analysis of randomized controlled trials. Prev Med 2009;48(1):9-19.

75. Halle M, Berg A, Baumstark MW, Keul J. Association of physical fitness with LDL and HDL subfractions in young healthy men. Int J Sports Med 1999;20:464-9.

76. O'Donovan G, McEneny J, Kearney EM, Owen A, Nevill AM, Woolf-May K, Bird SR. LDL particle size in habitual exercisers, lean sedentary men and abdominally obese sedentary men. Int J Sports Med 2007;28:644-9.

77. Houmard JA, Bruno NJ, Bruner RK, McCammon MR, Israel RG, Barakat HA. Effects of exercise training on the chemical composition of plasma LDL. Arterioscler Thromb 1994;14:325-30.

78. Szapary PO, Bloedon LT, Foster GD. Physical activity and its effects on lipids. Curr Cardiol Rep 2003;5:488-92.

79. Chan SY, Mancini GB, Burns S, Johnson FF, Brozic AP, Kingsbury K, Ignaszewski A, et al. Dietary measures and exercise training contribute to improvement of endothelial function and atherosclerosis even in patients given intensive pharmacologic therapy. J Cardiopulm Rehabil 2006;26:288-93.

80. Czajkowska A, Lutosławska G, Mazurek K, Ambroszkiewicz J, Zmijewski P. The relationship between physical activity and plasma homocysteine level in young men. Endokrynol Diabetol Chor Przemiany Materii Wieku Rozw 2008;14:177-80.

81. Margonis K, Fatouros IG, Jamurtas AZ, Nikolaidis MG, Douroudos I, Chatzinikolaou A, et al. Oxidative stress biomarkers responses to physical overtraining: implications for diagnosis. Free Radic Biol Med 2007;43:901-10. Erratum in: Free Radic Biol Med 2008;44:2058.

82. Chicco AJ, McCarty H, Reed AH, Story RR, Westerlind KC, Turner RT, Hayward R. Resistance exercise training attenuates alcohol-induced cardiac oxidative stress. Eur J Cardiovasc Prev Rehabil 2006;13:74-9.

83. Vincent HK, Bourguignon C, Vincent KR. Resistance training lowers exercise-induced oxidative stress and homocysteine levels in overweight and obese older adults. Obesity (Silver Spring) 2006;14:1921-30.

84. Steenge GR, Verhoef P, Greenhaff PL. The effect of creatine and resistance training on plasma homocysteine concentration in healthy volunteers. Arch Intern Med 2001;161:1455-6.

85. Liu-Ambrose T, Donaldson MG. Exercise and cognition in older adults: is there a role for resistance training programmes? Br J Sports Med 2009;43:25-7.

86. De Jong N, Chin A Paw MJ, de Groot LC, Rutten RA, Swinkels DW, Kok FJ, Van Staveren WA. Nutrientdense foods and exercise in frail elderly: effects on B vitamins, homocysteine, methylmalonic acid, and neuropsychological functioning. Am J Clin Nutr 2001;73:338-46.

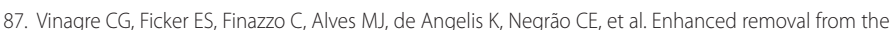
plasma of LDL-like nanoemulsion cholesteryl ester in trained men compared with sedentary healthy men. J Appl Physiol 2007;103:1166-71.

88. Silva JL, Vinagre CG, Morikawa AT, Maranhão RC. The effects of resistance exercise on plasma kinetics of an artificial nanoemulsion that binds to low-density lipoprotein receptors. World Congress of Cardiology 2008 - Circulation 2008;118:359-359.

89. Schjerve IE, Tyldum GA, Tjonna AE, Stolen T, Loennechen JP, Hansen HE, Wisloff U, et al. Both aerobic endurance and strength training programmes improve cardiovascular health in obese adults. Clin Sci (Lond) 2008;115:283-93. 